\title{
Sound power emitted by a pure-tone source in a reverberation room
}

\section{Jacobsen, Finn; Molares, Alfonso Rodriguez}

\section{Published in:}

Acoustical Society of America. Journal

Link to article, DOI:

$10.1121 / 1.3158918$

Publication date:

2009

Document Version

Publisher's PDF, also known as Version of record

Link back to DTU Orbit

Citation (APA):

Jacobsen, F., \& Molares, A. R. (2009). Sound power emitted by a pure-tone source in a reverberation room. Acoustical Society of America. Journal, 126(2), 676-684. https://doi.org/10.1121/1.3158918

\section{General rights}

Copyright and moral rights for the publications made accessible in the public portal are retained by the authors and/or other copyright owners and it is a condition of accessing publications that users recognise and abide by the legal requirements associated with these rights.

- Users may download and print one copy of any publication from the public portal for the purpose of private study or research.

- You may not further distribute the material or use it for any profit-making activity or commercial gain

- You may freely distribute the URL identifying the publication in the public portal

If you believe that this document breaches copyright please contact us providing details, and we will remove access to the work immediately and investigate your claim. 


\title{
Sound power emitted by a pure-tone source in a reverberation room $^{\text {a) }}$
}

\author{
Finn Jacobsen ${ }^{\text {b) }}$ \\ Acoustic Technology, Department of Electrical Engineering, Technical University of Denmark, Building 352, \\ DK-2800 Kgs. Lyngby, Denmark \\ Alfonso Rodríguez Molares \\ ETSE Telecomunicación, Universidade de Vigo, Campus Lagoas-Marcosende, E-36310 Vigo, Spain
}

(Received 18 February 2009; revised 5 May 2009; accepted 26 May 2009)

Energy considerations are of enormous practical importance in acoustics. In "energy acoustics," sources of noise are described in terms of the sound power they emit, the underlying assumption being that this property is independent of the particular environment where the sources are placed. However, it is well known that the sound power output of a source emitting a pure tone or a narrow band of noise actually varies significantly with its position in a reverberation room at low frequencies, and even larger variations occur between different rooms. The resulting substantial uncertainty in measurements of sound power as well as in predictions based on knowledge of sound power is one of the fundamental limitations of energy acoustics. The existing theory for this phenomenon is fairly complicated and has only been validated rather indirectly. This paper describes a far simpler theory and demonstrates that it gives predictions in excellent agreement with the established theory. The results are confirmed by experimental results as well as finite element calculations. (c) 2009 Acoustical Society of America. [DOI: 10.1121/1.3158918]

PACS number(s): 43.55.Cs, 43.58.Bh [AJZ]

Pages: 676-684

\section{INTRODUCTION}

It has been known for many years that sound power emitted by a stationary sound source in a reverberant room depends on the particulars of the room (shape, size, and damping) and the position of the source. The variations are relatively small for sources of broad band noise but fairly substantial for sources that emit pure tones, in particular below the Schroeder frequency. This implies a fundamental contribution to the measurement uncertainty in any sound power measurement that takes place in a non-anechoic room irrespective of whether the diffuse-field method or the sound intensity method is used and, of course, to a similar uncertainty in any prediction of the sound pressure level generated by the source in another room calculated on the basis of the sound power of the source (even if this quantity has been measured under free-field conditions and is very close to the "true" free-field sound power). For pure-tone sources, this fundamental component of the uncertainty is much larger than all other contributions. The only way of reducing it is to determine the sound power at different positions in different rooms or in a room that can be changed, e.g., with a large rotating vane.

\footnotetext{
"Portions of this work were presented in "The sound power of sources in reverberant surroundings," Proceedings of NOVEM 2009, Cambridge, England, April 2009.

b) Author to whom correspondence should be addressed. Electronic mail: fja@elektro.dtu.dk
}

\section{A BRIEF DESCRIPTION OF THE ESTABLISHED THEORY}

The existing theory is essentially due to Lyon ${ }^{1}$ and Davy $^{2}$ but is later modified in accordance with more recent findings by Weaver. ${ }^{3}$ Lyon's approach was based on the analytical Green's function in a rectangular room (and in a point-driven plate), which is a modal sum. Replacing modal sums by integrals and assuming that the modal frequencies have a Poisson distribution (i.e., are distributed independently), he derived the following expression for the normalized standard deviation of the sound power output of a monopole emitting a pure tone in a room,

$$
\varepsilon\left\{P_{a}\right\}=\frac{1}{\sqrt{M_{s}}}\left(\frac{3}{2}\right)^{3 / 2},
$$

where $M_{s}$ is the statistical modal overlap,

$$
\begin{aligned}
M_{s} & =n(f) B_{s}=\frac{4 \pi V f^{2}}{c^{3}} \frac{1}{2 \tau}=\frac{12 \pi \ln (10) V f^{2}}{T_{60} c^{3}}=\frac{S \operatorname{Re}\{\beta\} k^{2}}{\pi} \\
& =\frac{A k^{2}}{8 \pi},
\end{aligned}
$$

in which $n(f)$ is the modal density (in modes per hertz), $B_{s}$ is the statistical modal bandwidth (in hertz), $V$ is the volume of the room, $f$ is the frequency, $c$ is the speed of sound, $\tau$ is the modal time constant, $T_{60}$ is the corresponding reverberation time, $\beta$ is the wall admittance normalized by the characteristic impedance of air, $S$ is the surface area of the room, $k$ is the wave number, and $A$ is the total absorption area of the room. (In this expression, one-dimensional (1D) and twodimensional (2D) modes have been ignored.) Lyon used the 
equivalent noise modal bandwidth, which is half the statistical bandwidth. Although Lyon's expression at that time was largely interpreted as the source position variance, it is clear that since his statistical method involved different distributions of the modal frequencies the expression is in effect a theoretical estimate of the normalized ensemble standard deviation, with variation over rooms-a quantity that is evidently of fundamental importance for the uncertainty but difficult to measure.

Twelve years later, Davy ${ }^{2}$ extended Lyon's theory by deriving a more general expression of the power transmission function averaged over multiple source and receiver positions. Assuming a "nearest neighbor" distribution of the modal frequencies, he found a normalized ensemble standard deviation of the output power (or the real part of the radiation impedance) of

$$
\varepsilon\left\{P_{a}\right\}=\frac{1}{\sqrt{M_{s}}}\left(\left(\frac{3}{2}\right)^{3}-\frac{1}{2}\right)^{1 / 2}
$$

for high modal overlap, ignoring 1D and 2D modes in a rectangular room. (No simple expression in closed form was derived for the case of low modal overlap.) Some experimental results from measurements of power transmission functions in a very large $\left(600 \mathrm{~m}^{3}\right)$ room were presented. However, Davy's main issue in this paper was to demonstrate that a very different theory derived by Waterhouse ${ }^{4}$ was incorrect, and this he certainly did. The assumption of the modal frequencies having a nearest neighbor distribution rather than being distributed independently came from evidence of a "repulsion" effect between modal frequencies already anticipated by Lyon. ${ }^{1}$ The modal frequencies seem to repel each other, and thus their distribution is not completely random but closer to the average density than one might have expected.

Some years later, Davy ${ }^{5}$ discussed possible improvements of his theory, but in this paper he was essentially concerned with the variance of random noise passed through a reverberation room and measured using a finite averaging time.

In the late 1980s, Weaver ${ }^{3}$ discussed Davy's theory and suggested replacing his $K$ [the term $(3 / 2)^{3}$ in Eq. (3)] with 3, "which is appropriate for a Gaussian distribution of amplitudes and based on vague arguments invoking the central limit theorem." At that time, it had been established that modal frequencies tend to exhibit "spectral rigidity" or "long range repulsions" in accordance with the random matrix theory of Gaussian orthogonal ensembles. ${ }^{6}$ This seems to be generally accepted now. ${ }^{7}$ A value of $K=3$ has also been favored by Lobkis et al. ${ }^{8}$

In 1990, Davy ${ }^{9}$ modified his theory so as to take account of the Gaussian orthogonal ensemble modal frequency spacing. Equation (3) now became

$$
\varepsilon\left\{P_{a}\right\}=\frac{1}{\sqrt{M_{s}}}\left(\left(\frac{3}{2}\right)^{3}-1\right)^{1 / 2} .
$$

This expression was further discussed in yet another congress paper by Davy ${ }^{10}$ from the late 1990s. It can easily be modified so as to take account of 1D and 2D modes. How- ever, it is not completely clear from these papers whether its validity is restricted to high modal overlap, as Eq. (3).

Various consequences of Davy's, ${ }^{2,9,10}$ original and modified theory have been examined experimentally, but there is very little experimental evidence in direct support of Eq. (4).

\section{AN ALTERNATIVE THEORY}

The alternative theory presented in what follows is far simpler than the theory briefly described in the foregoing and does not even take account of the phenomenon of modes. It was derived independently by Jacobsen ${ }^{11}$ and Pierce ${ }^{12}$ about 30 years ago.

The sound field in a reverberant room is modeled as an infinite sum of plane waves,

$$
\begin{aligned}
p_{\text {rev }}(\mathbf{r}) & =\lim _{N \rightarrow \infty} \frac{1}{\sqrt{N}} \sum_{n=1}^{N} A_{n} e^{j\left(\omega t+\mathbf{k}_{n} \cdot \mathbf{r}\right)} \\
& =\lim _{N \rightarrow \infty} \frac{1}{\sqrt{N}} \sum_{n=1}^{N}\left|A_{n}\right| e^{j\left(\omega t+\varphi_{n}+\mathbf{k}_{n} \cdot \mathbf{r}\right)},
\end{aligned}
$$

where the phase angles $\varphi_{n}$ are uniformly distributed between 0 and $2 \pi$, the amplitudes $\left|A_{n}\right|$ have an arbitrary distribution, the wave number vectors $\mathbf{k}_{n}$ are uniformly distributed over all angles of incidence (corresponding to a sinusoidal distribution of the polar angles and a uniform distribution of the azimuth angles), and $\mathbf{r}$ is the observation point. This stochastic pure-tone diffuse-field interference model was originally developed by Waterhouse. ${ }^{13}$

The sound field at the source position $\left(\mathbf{r}_{0}\right)$ may be regarded as the sum of the direct field and the reverberant field, and therefore the radiation impedance is the sum of the freefield radiation impedance and the complex ratio of the sound pressure associated with the reverberant field at the source position and the volume velocity of the source,

$$
Z_{r}=\frac{\rho c k^{2}}{4 \pi}+\frac{j k \rho c}{4 \pi a}+\frac{p_{\mathrm{rev}}\left(\mathbf{r}_{0}\right)}{Q e^{j \omega t}},
$$

where $\rho$ is the density of air, $a$ is the radius of the monopole that generates the sound field (modeled as a small pulsating sphere), and $Q$ is its volume velocity. (As pointed out by Lyon, ${ }^{1}$ the imaginary part of the free-field radiation impedance diverges as the radius of the sphere goes toes to zero.) All phases are equally probable in the reverberant part of the sound field, which leads to the conclusion that on the average the monopole emits its free-field sound power output,

$$
E\left\{P_{a}\right\}=\frac{|Q|^{2}}{2} E\left\{\operatorname{Re}\left\{Z_{r}\right\}\right\}=P_{a \text {,free field }}=\frac{\rho c k^{2}|Q|^{2}}{8 \pi} .
$$

However, because of the third term in Eq. (6) the actual sound power output of the source varies between different outcomes of the stochastic process; i.e., it varies with the source position and it varies from room to room. The corresponding variance can be calculated as follows: 


$$
\begin{aligned}
\sigma^{2}\left\{P_{a}\right\} & =\frac{|Q|^{4}}{4} \sigma^{2}\left\{\operatorname{Re}\left\{Z_{r}\right\}\right\}=\frac{|Q|^{4}}{4} \sigma^{2}\left\{\operatorname{Re}\left\{\frac{p_{\text {rev }}\left(\mathbf{r}_{0}\right)}{Q e^{j \omega t}}\right\}\right\} \\
& =\frac{|Q|^{2}}{4} E\left\{\left|p_{\text {rev }}\left(\mathbf{r}_{0}\right)\right|^{2} \cos ^{2}\left(\phi\left(\mathbf{r}_{0}\right)\right)\right\} \\
& =\frac{|Q|^{2}}{8} E\left\{\left|p_{\text {rev }}\left(\mathbf{r}_{0}\right)\right|^{2}\right\},
\end{aligned}
$$

where $\phi$ is the phase angle of $p_{\text {rev }}\left(\mathbf{r}_{0}\right)$, which is a random variable uniformly distributed between 0 and $2 \pi$. The expectation of the mean square reverberant pressure can be approximated by its spatial average, which is related to the actual sound power emitted by the monopole and the total absorption area of the room by the energy balance equation, ${ }^{12}$

$$
E\left\{\left|p_{\text {rev }}\right|^{2}\right\} \simeq\left\langle\left|p_{\text {rev }}\right|^{2}\right\rangle=\frac{8 \rho c}{A} P_{a},
$$

where \langle\rangle indicates a spatial average. Equation (8) now becomes

$$
\sigma^{2}\left\{P_{a}\right\} \simeq \frac{|Q|^{2}}{8} \frac{8 \rho c}{A} P_{a} \simeq E^{2}\left\{P_{a}\right\} \frac{8 \pi}{A k^{2}}=\frac{E^{2}\left\{P_{a}\right\}}{M_{s}},
$$

where use has been made of Eq. (7) and $P_{a}$ has been approximated by its expectation. Normalizing with the free-field sound power finally gives

$$
\varepsilon\left\{P_{a}\right\} \simeq \frac{1}{k} \sqrt{\frac{8 \pi}{A}}=\frac{1}{\sqrt{M_{s}}} .
$$

It is interesting to note that this expression is identical to Davy's [Eq. (4)] except for a constant factor, and it is quite surprising that the modal overlap enters into a theory that does not even operate with the concept of a mode. The expression can, of course, easily be extended so as to include the effect of 1D and 2D modes in a rectangular room. Note, however, that no assumptions about the shape (or size) of the room have been made.

Expression (11) does not take account of the phenomenon of "weak Anderson localization," also known as coherent backscattering, ${ }^{7,14,15}$ according to which there is a concentration of the reverberant sound field at the source position. Such a phenomenon was already predicted on the basis of Poisson statistics in Lyon's paper. ${ }^{1}$ According to Langley and Cotoni, ${ }^{7}$ the now generally accepted assumption of Gaussian orthogonal ensemble statistics leads to a "concentration factor" for the mean square reverberant field that approaches 3 for $M_{s} \rightarrow 0$ and 2 for $M_{s} \rightarrow \infty$. This can be expected to modify Eq. (11) to

$$
\varepsilon\left\{P_{a}\right\}=\sqrt{\frac{F\left(M_{s}\right)}{M_{s}}},
$$

\begin{tabular}{|c|c|c|c|}
\hline Description of room & $\begin{array}{l}\text { Volume } \\
\left(\mathrm{m}^{3}\right)\end{array}$ & $\begin{array}{l}\text { Reverberation } \\
\text { time at } \\
500 \mathrm{~Hz} \\
\text { (s) }\end{array}$ & $\begin{array}{c}\text { Schroeder } \\
\text { frequency } \\
(\mathrm{Hz})\end{array}$ \\
\hline Small lightly damped room & 40 & 2.3 & 460 \\
\hline Small heavily damped room & 40 & 0.8 & 290 \\
\hline Large reverberation room & 245 & 5.7 & 300 \\
\hline Very large reverberation room & 500 & 5.8 & 220 \\
\hline Large strongly damped hall & 650 & 1.1 & 90 \\
\hline
\end{tabular}

where the concentration factor $F$ is a function that goes smoothly from 3 to 2 as the modal overlap increases. It is worth noting that Eqs. (4) and (12) are identical if $K=3$ (as suggested in Refs. 3 and 8) and $F=2$.
TABLE I. Reverberation rooms used in the experiments.

\section{EXPERIMENTAL AND NUMERICAL RESULTS}

To examine the validity of the established theory and the alternative simpler one, some experiments have been carried out in different reverberation rooms, but since it is impossible in practice to determine statistical properties associated with an ensemble of rooms the experiments are supplemented by finite element calculations.

\section{A. Experimental results}

A "volume velocity source," Brüel \& Kjær (B\&K) 4295 (a tube with two matched quarter-inch microphones driven by a loudspeaker), can also be used for measuring the radiation impedance of the small opening of the tube, which is related to the frequency response between the two microphone signals (see Appendix A). This method has been used for measuring the radiation impedance at 24 positions in four different rooms, one of which in two different conditions (see Table I). Figure 1 shows the reverberation time of the rooms measured in one-third octave bands with a B\&K "PULSE" analyzer using interrupted noise.

The frequency response between the microphone signals from the volume velocity source was measured with the same analyzer in fast Fourier transform mode with 6400

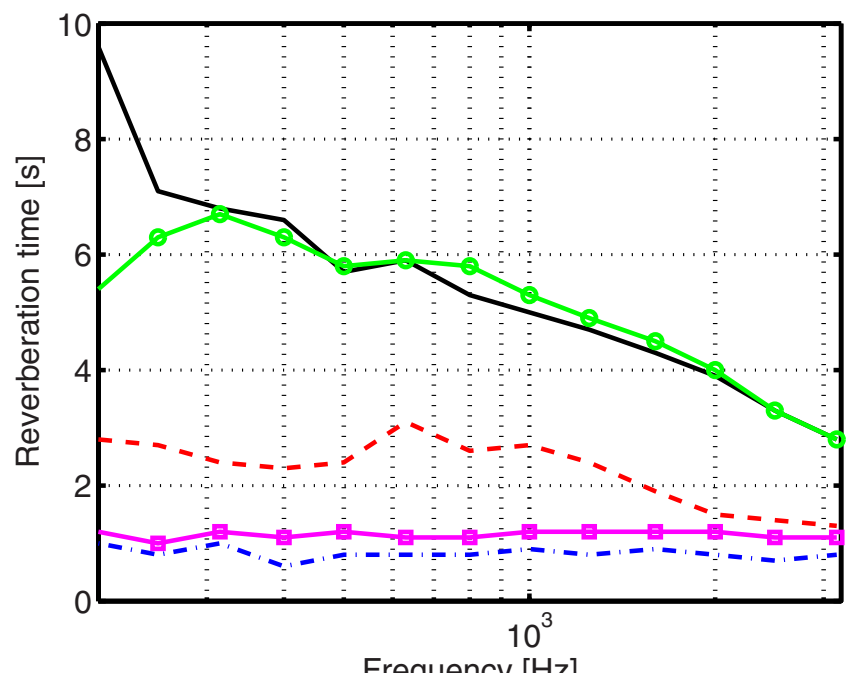

Frequency $[\mathrm{Hz}]$

FIG. 1. (Color online) Reverberation time of the test rooms. Solid line: large reverberation room; solid line with circles: very large reverberation room; dashed line: small lightly damped room; solid line with squares: large heavily damped hall; dash-dotted line: small heavily damped room.

F. Jacobsen and A. R. Molares: Source power in reverberation rooms 

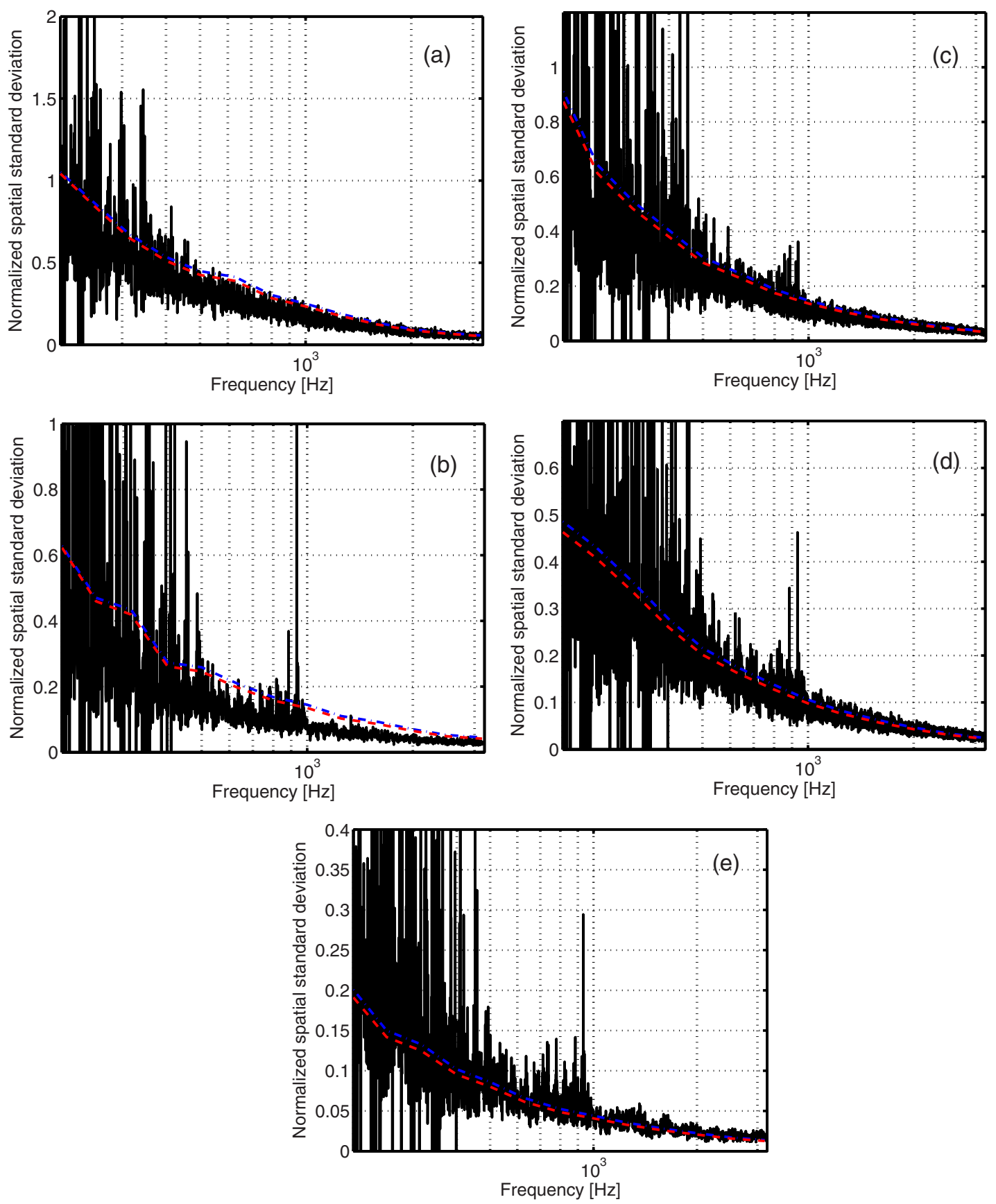

FIG. 2. (Color online) Normalized spatial standard deviation of sound power output in (a) small lightly damped small room, (b) small heavily damped room, (c) large reverberation room, (d) very large reverberation room, and (e) large heavily damped hall. Solid line: measured standard deviation; dash-dotted line: prediction based on Eq. (4); dashed line: prediction based on Eq. (12) with $F=2$.

spectral lines and a resolution of $0.5 \mathrm{~Hz}$. The source was driven with synchronized pseudorandom noise generated by the PULSE analyzer, and a uniform time window was used. This corresponds to measuring at 6400 independent discrete frequencies. A similar technique has been used by Baade and Maling ${ }^{16}$ in "qualification" of reverberation rooms. Measuring the radiation impedance of the volume velocity source directly is extremely efficient compared with measuring the sound power output with a conventional method using many source and receiver positions, but it should be mentioned that this technique makes heavy demands on the equipment as reflected in many (meaningless) negative estimates at low frequencies (see Appendix A).
Figure 2 compares the normalized spatial standard deviation of the real part of the radiation impedance observed in the five rooms with predictions based on Davy's Eq. (4) and the new expression, Eq. (12), with $F=2$ because the modal overlap is large except at very low frequencies. Since all the rooms are essentially rectangular, Eqs. (4) and (12) have been modified to take account also of $2 \mathrm{D}$ and $1 \mathrm{D}$ modes, although the effect of this modification is almost negligible. It is interesting that the two fundamentally different theories give practically identical predictions. At low frequencies, the measured spatial standard deviations fluctuate enormously with the frequency. This is not unexpected since the spatial standard deviation at low modal overlap depends 

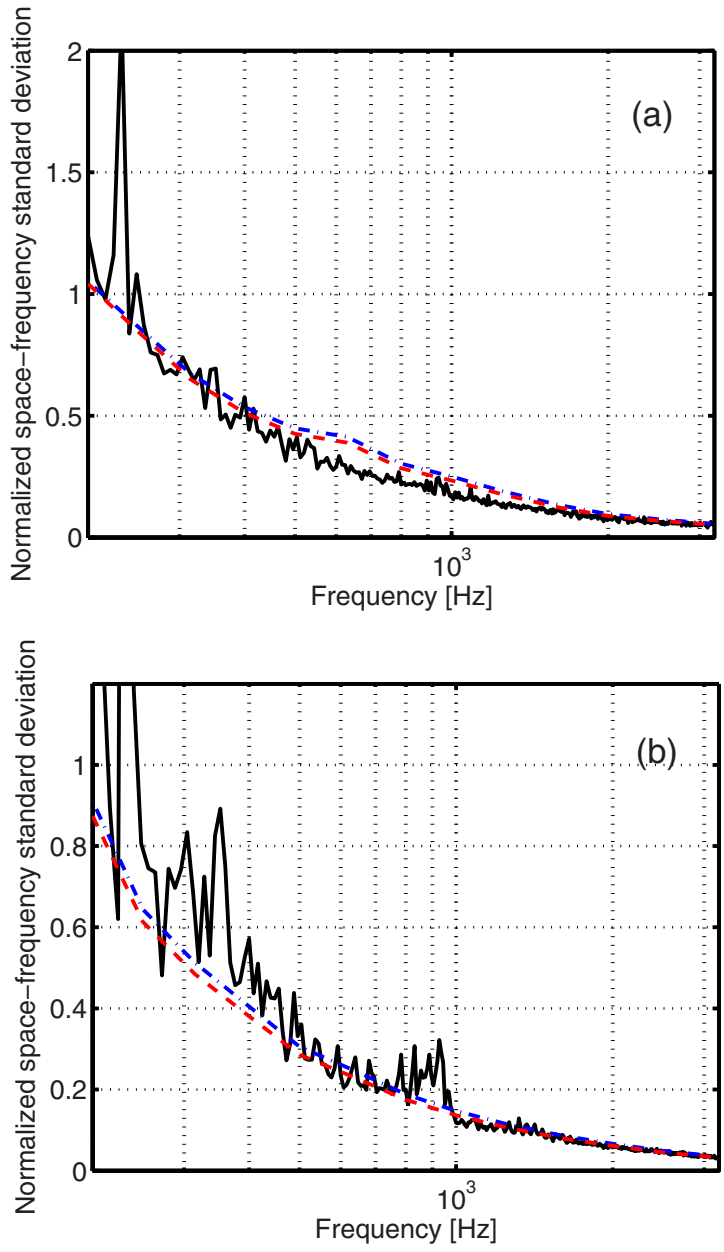

FIG. 3. (Color online) Normalized space-frequency standard deviation of sound power output in (a) small lightly damped room and (b) large reverberation room. Solid line: measured standard deviation; dash-dotted line: prediction based on Eq. (4); dashed line: prediction based on Eq. (12) with $F=2$.

strongly on whether one single mode dominates or whether several modes contribute to the sound field. The spatial standard deviation tends to be slightly lower than the predicted ensemble standard deviation except in the large hall, but it certainly has the same tendency as the predictions in all cases.

Figure 3 shows the corresponding space-frequency standard deviation in two of the five rooms, calculated from variations in the same data with respect not only to source positions but also to 16 neighboring frequencies (corresponding to a band of $8 \mathrm{~Hz}$ ). The latter is an attempt to estimate the ensemble standard deviation. There is fair, if not perfect, agreement between predictions and experimental results.

\section{B. Finite element calculations}

An alternative approach involves calculating the ensemble and spatial sound power variance numerically using the finite element method (FEM). With a FEM model of a room enclosing a point source, the sound power can be calculated simply by performing the integration of the normal component of the sound intensity along the boundaries. This integration can readily be performed by means of functions available in most FEM packages. If the position of the point source is varied, the spatial variance in the room can be obtained. If, in addition, the calculation is carried out in a set of rooms with different shapes, and thus with different modal overlaps, the ensemble variance of sound power can be achieved by computing the variance of sound power obtained for all source positions in all tested rooms. However, the calculation of the ensemble variance must be performed directly in terms of the modal overlap. Since this quantity depends on the total absorption area of the room, or its surface area and wall impedance [see Eq. (2)], the modal overlaps of the various rooms may differ. If one would try to compute the variance of all points for a certain value of the modal overlap, only values of a single room would be available. The solution chosen here is to divide the modal overlap axis into bands. Performing the calculation in such modal overlap intervals makes it possible to estimate the ensemble variance, provided that there are enough results from different rooms in the intervals.

Two sets of calculations were carried out: threedimensional (3D) calculations that can be compared directly with the experimental data and 2D calculations that make it possible to increase the frequency range. The equations for 2D are given in Appendix B. For simplicity, the rooms were rectangular. All calculations were made using the FEM software package COMSOL 3.4, with which one can specify excitation by a monopole at a given position. To guarantee a low numerical pollution, the quantity $k h / 2 p$ (in which $h$ is the maximum size of the elements and $p$ is their order) was kept less than $0.5 .^{17}$ In both cases, the sound source was placed randomly in the room, provided that it was at least $0.4 \mathrm{~m}$ away from the walls. For the $3 \mathrm{D}$ calculations the room dimensions were changed from $2 \times 5 \times 4.27$ to $4 \times 3$ $\times 3.56 \mathrm{~m}^{3}$, and for the $2 \mathrm{D}$ calculations the dimensions of the "room" were changed from $2 \times 7.2$ to $4 \times 3.6 \mathrm{~m}^{2}$. In all cases, the walls were locally reacting with an impedance of $87000 \mathrm{~Pa} \mathrm{~s} / \mathrm{m}$. The 3D calculations were carried out from 50 to $300 \mathrm{~Hz}$ with a frequency step of $1 \mathrm{~Hz}$, and the ensemble variance was calculated in modal overlap bands with a width of 0.09 . The $2 \mathrm{D}$ calculations were carried out in the same frequency range with a frequency step of $12.5 \mathrm{~Hz}$ and modal overlap intervals of 0.02 .

Figure 4(a) compares the calculated ensemble standard deviation of the sound power with respect to 50 different 3D rooms and 50 source positions with predictions calculated using Eqs. (4) and (12), and Fig. 4(b) does the same for the $2 \mathrm{D}$ case, in both cases with $F=2$ as well as with $F=3$ in Eq. (12). In the $3 \mathrm{D}$ case, the two theories lead to practically identical predictions with $F=2$, but the agreement with the FEM results is better, in fact almost perfect, with $F=3$. This agrees with Langley and Cotoni's ${ }^{7}$ prediction of a concentration factor of 3 at low modal overlap. In the 2D case, Eq. (4) underestimates and Eq. (12) overestimates the observed standard deviations with $F=3$, whereas Eq. (12) agrees extremely well with the FEM results with $F=2$.

\section{DISCUSSION}

It should be emphasized that the underlying theories of Eqs. (4) and (12) are quite different. Davy's model is based 

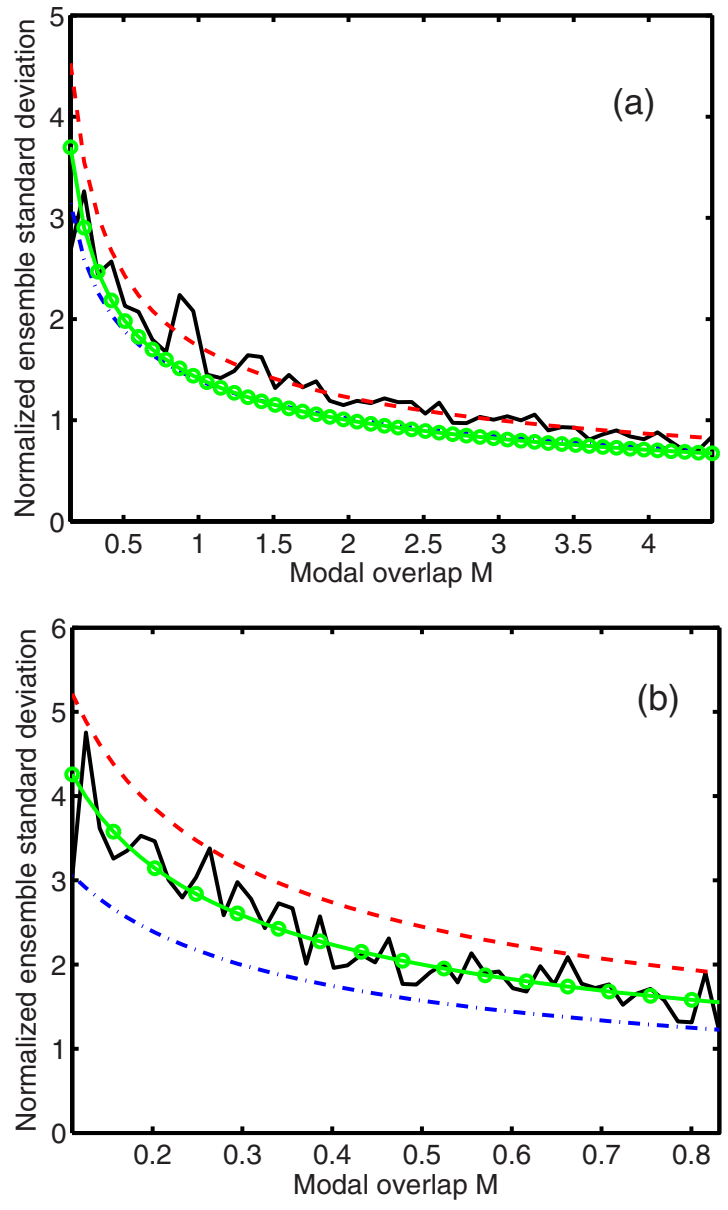

FIG. 4. (Color online) Normalized ensemble standard deviation of sound power output in (a) 3D rooms and (b) 2D rooms. Solid line: FEM results; dash-dotted line: prediction based on Eq. (4); solid line with circles: prediction based on Eq. (12) with $F=2$; dashed line: prediction based on Eq. (12) with $F=3$.

on the analytical Green's function in a rectangular room, makes use of the concept of impedance, and requires knowledge of mode shapes and the modal density and distribution. The alternative theory does not even recognize the concept of modes, and the losses of the rooms are described in terms of the absorption area. Thus the almost perfect agreement between the predictions (in the 3D case) is surprising. Note, however, that the factor of $F$ has been derived in the literature using a modal approach. ${ }^{7,14}$ The free wave model cannot possibly take account of the coherent backscattering effect since the waves might be generated by independent sources.

Both the established theory and the alternative one should be taken with a grain of salt at very low frequencies. For example, it is obvious that at extremely low frequencies, well below the lowest modal frequency, both theories predict a huge variance, whereas in reality the spatial source position variance is zero and the variation between rooms is only related to their different volumes. The spatial source position variance is also limited in the single mode case $(19 / 8,5 / 4$, and $1 / 2$ for $3 \mathrm{D}, 2 \mathrm{D}$, and $1 \mathrm{D}$ modes in a rectangular room, respectively ${ }^{18}$ ).

At somewhat higher frequencies but below the Schroeder frequency, the spatial variance is strongly affected by whether the frequency coincides with a modal frequency. However, above the Schroeder frequency where the modal overlap is high, one would expect the same statistics with respect to room, position, and frequency. In other words, merely varying the source position can be expected to lead to the full ensemble variance in this frequency range because many modes are excited at all frequencies, and this ergodicity seems to be confirmed by the experimental results, although Schroeder's original "large room frequency," which corresponds to twice the established Schroeder frequency, ${ }^{19}$ is perhaps more adequate.

\section{CONCLUSION}

Experimental and numerical results confirm the wellknown observation that there is a substantial uncertainty in sound power measurements of pure-tone sources in nonanechoic rooms at low frequencies. The associated standard deviation is inversely proportional to the square root of the modal overlap, that is, essentially proportional to the square root of the ratio of the reverberation time to the volume of the room and inversely proportional to the frequency.

Experimental results obtained in four very different rooms, one of which in two different damping conditions, confirm that the sound power output of the monopole exhibits the same statistical fluctuations with respect to changes in the source position and changes in the frequency somewhat above the Schroeder frequency.

An extremely simple, very general model based on sound waves arriving with random phases from random directions has, surprisingly, been found to give predictions of the ensemble standard deviation of the sound power of a monopole in almost perfect agreement with the predictions of the established, far more complicated theory. These predictions are in fairly good agreement with experimental observations and in excellent agreement with the results of finite element calculations in three dimensions. The alternative model indirectly confirms the phenomenon known as weak Anderson localization in reverberation rooms and seems also to confirm that the concentration factor, which describes the local increase in the reverberant sound energy at the source position, is 3 at low modal overlap and 2 at higher modal overlap.

In $2 \mathrm{D}$ rooms, the results are slightly different. The general tendencies are the same, but in this case there is a systematic difference between the two theories, and the alternative one is in best agreement with the finite element calculations if the assumed concentration factor is 2 .

\section{ACKNOWLEDGMENTS}

The first author would like to thank John Davy and Richard Weaver for stimulating discussions during the Acoustics'08 meeting in Paris in June/July 2008.

\section{APPENDIX A: EXPERIMENTAL TECHNIQUE}

A method of measuring the volume velocity of an "experimental monopole" has been described in Refs. 20 and 21. The method is based on a tube with two matched quarterinch microphones driven by a loudspeaker at the other end, 


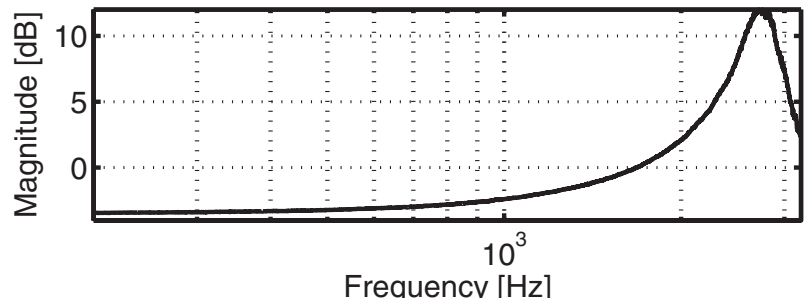

Frequency $[\mathrm{Hz}]$

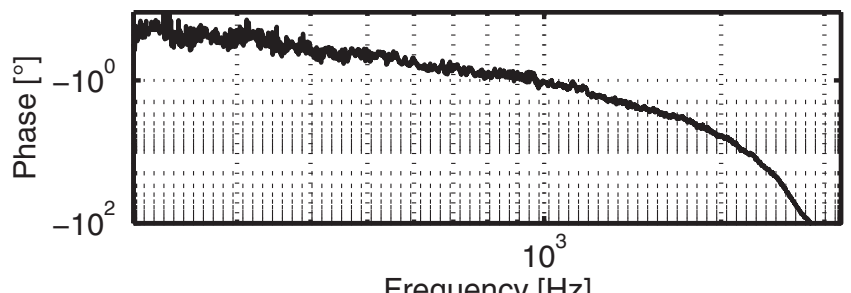

Frequency $[\mathrm{Hz}]$

FIG. 5. Magnitude and phase of a frequency response in the tube, $H_{A B}$.

and this device, B\&K 4295, can also be used for measuring the radiation impedance of the small opening of the tube. In the frequency range where it can be assumed that only plane waves propagate in the tube, the volume velocity at the opening of the tube can readily be shown to be

$$
Q=\frac{S_{t}}{\rho c} \cdot \frac{p_{A} \cos k l-p_{B} \cos [k(l+\Delta l)]}{j \sin k \Delta l},
$$

where $S_{t}$ is the cross-sectional area of the tube, $p_{A}$ and $p_{B}$ are the two pressure signals, $\Delta l$ is the distance between the mi-

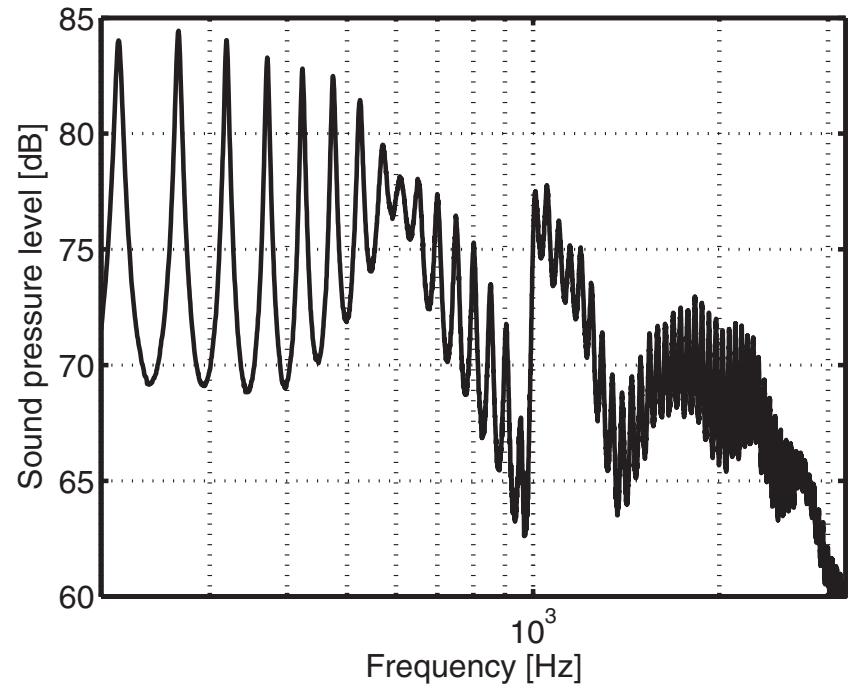

FIG. 6. Power spectrum of the sound pressure measured near the opening of the tube.

crophones, and $l$ is the distance between the opening and the microphone nearest the opening. The sound pressure at the opening is

$$
p=\frac{-p_{A} \sin k l+p_{B} \sin [k(l+\Delta l)]}{\sin k \Delta l} .
$$

It now follows that the real part of the radiation impedance is

$$
\operatorname{Re}\left\{Z_{r}\right\}=\operatorname{Re}\left\{\frac{p}{Q}\right\}=-\frac{\rho c}{S_{t}} \frac{\operatorname{Im}\left\{p_{A}^{*} p_{B}\right\} \sin (k \Delta l)}{\left|p_{A}\right|^{2} \cos ^{2}(k l)+\left|p_{B}\right|^{2} \cos ^{2}[k(l+\Delta l)]-2 \operatorname{Re}\left\{p_{A}^{*} p_{B}\right\} \cos (k l) \cos [k(l+\Delta l)]} .
$$

Expressed in terms of the frequency response between the two pressure signals, this becomes

$$
\operatorname{Re}\left\{Z_{r}\right\}=-\frac{\rho c}{S_{t}} \frac{\operatorname{Im}\left\{H_{A B}\right\} \sin (k \Delta l)}{\cos ^{2}(k l)+\left|H_{A B}\right|^{2} \cos ^{2}[k(l+\Delta l)]-2 \operatorname{Re}\left\{H_{A B}\right\} \cos (k l) \cos [k(l+\Delta l)]} .
$$

The measurement is not without problems, and at low frequencies some estimates of the real part of the radiation impedance are negative, which is why it was chosen to present the results only from $200 \mathrm{~Hz}$ and upward. The pressure-intensity index, a quantity well known from the literature on sound intensity measurements with conventional sound intensity probes based on two matched pressure microphones, ${ }^{22}$ can easily be calculated from the frequency response between the microphone signals, ${ }^{23}$

$10 \log \left(\frac{p_{\text {mean }}^{2} /(\rho c)}{I}\right)=10 \log \left(\frac{\left(1+\left|H_{A B}\right|^{2}+2 \operatorname{Re}\left\{H_{A B}\right\}\right) / 4}{-\operatorname{Im}\left\{H_{A B}\right\} /(k \Delta l)}\right)$,

and is about $13 \mathrm{~dB}$ below $1 \mathrm{kHz}$, indicating rather harsh sound field conditions. However, the microphones of B\&K
4295 are matched so well that this is not a very serious problem, and an attempt to compensate for possible phase and amplitude mismatch (using the "sensor-switching technique" suggested by Chung and Blaser ${ }^{24}$ ) did not improve conditions.

Figure 5 shows an example of a frequency response $\left(H_{A B}\right)$ measured in the large hall. Note that the phase angle of this function varies between about $-0.2^{\circ}$ at $200 \mathrm{~Hz}$ and about $-1^{\circ}$ at $1 \mathrm{kHz}$. Inspection of Eq. (A4) shows that the information about the real part of the radiation impedance (or the emitted sound power) is associated with the imaginary part of the frequency response, which is obviously very small below $1 \mathrm{kHz}$, and the information about how the emitted sound power varies from position to position is hidden in minute fluctuations in this very small imaginary part of the frequency response. There are strong resonances in the loudspeaker cavity and tube, as can be seen in Fig. 6, which 
shows the spectrum of the microphone signal nearest the opening of the tube. These resonances should obviously not affect the radiation impedance at the outlet of the tube. However, it is believed that the most serious source of error in the experimental results presented in Sec. IV is due to numerical problems in the signal processing caused by the imaginary part of the frequency response being several hundred times smaller than the real part. Thus the resonances may nevertheless have had some effects on the estimated radiation impedance.

\section{APPENDIX B: SOUND POWER IN 2D ROOMS}

By analogy with Eq. (5), a 2D diffuse sound field can be modeled as an infinite sum of plane waves coming from all angles of incidence (from 0 to $2 \pi$ ) and having random phases. Such a sound field might conceivably be generated by an infinite pulsating line source in an infinitely long room. The sound intensity that is incident on the walls of such a room is

$$
I_{\text {inc }}^{\prime}=\frac{1}{2 \pi} \int_{-\pi / 2}^{\pi / 2} \frac{E\left\{\left|p_{\mathrm{rev}}\right|^{2}\right\}}{2 \rho c} \cos \theta d \theta=\frac{E\left\{\left|p_{\mathrm{rev}}\right|^{2}\right\}}{2 \pi \rho c},
$$

and thus the sound power per unit length emitted by the source and absorbed by the walls is

$$
P_{a}^{\prime} \simeq \frac{E\left\{\left|p_{\mathrm{rev}}\right|^{2}\right\}}{2 \pi \rho c} A^{\prime},
$$

where $A^{\prime}$ is the absorption area of the walls of the room per unit length. On average, the sound power emitted by the line source equals its free-field sound power, ${ }^{25}$

$$
P_{a}^{\prime}=\frac{\left|Q^{\prime}\right|^{2}}{2} \operatorname{Re}\left\{Z_{r}^{\prime}\right\}=\frac{\rho c k\left|Q^{\prime}\right|^{2}}{8},
$$

where $Q^{\prime}$ is the volume velocity per unit length and $Z_{r}^{\prime}$ is the radiation impedance. However, because of the reverberant, diffuse sound field, the sound power output varies from position to position and from room to room. The corresponding variance is

$$
\begin{aligned}
\sigma^{2}\left\{P_{a}^{\prime}\right\} & =\frac{\left|Q^{\prime}\right|^{4}}{4} \sigma^{2}\left\{\operatorname{Re}\left\{Z_{r}^{\prime}\right\}\right\}=\frac{\left|Q^{\prime}\right|^{4}}{4} \sigma^{2}\left\{\operatorname{Re}\left\{\frac{p_{\mathrm{rev}}}{Q^{\prime} e^{j \omega t}}\right\}\right\} \\
& =\frac{\left|Q^{\prime}\right|^{2}}{4} E\left\{\left|p_{\mathrm{rev}}\right|^{2} \cos ^{2} \varphi\right\}=\frac{\left|Q^{\prime}\right|^{2}}{8} E\left\{\left|p_{\mathrm{rev}}\right|^{2}\right\} \\
& \simeq \frac{\left|Q^{\prime}\right|^{2}}{8} \frac{2 \pi \rho c}{A^{\prime}} E\left\{P_{a}^{\prime}\right\}=E^{2}\left\{P_{a}^{\prime}\right\} \frac{2 \pi}{A^{\prime} k} .
\end{aligned}
$$

If the source is suddenly turned off, the sound energy in the room decays with a time constant that follows from simple energy balance considerations,

$$
\tau^{\prime}=\frac{E_{a}^{\prime}}{P_{a}^{\prime}}=\frac{S^{\prime}}{2 L^{\prime} c \operatorname{Re}\left\{\beta^{\prime}\right\}}=\frac{\pi S^{\prime}}{c A^{\prime}},
$$

where $S^{\prime}=l_{x} \cdot l_{y}$ is the cross-sectional area of the infinitely long room, $L^{\prime}=2\left(l_{x}+l_{y}\right)$, and $\beta^{\prime}$ is the normalized wall admittance. The modal density in modes per hertz is

$$
n^{\prime}(f)=\frac{2 \pi S^{\prime} f}{c^{2}}
$$

and the statistical modal overlap is

$$
M_{s}^{\prime}=\frac{n^{\prime}(f)}{2 \tau^{\prime}}=\frac{A^{\prime} k}{2 \pi} .
$$

Combining Eqs. (B4) and (B7) finally shows that the normalized standard deviation of the emitted sound power has exactly the same form in $2 \mathrm{D}$ as in $3 \mathrm{D}$,

$$
\varepsilon\left\{P_{a}^{\prime}\right\}=\frac{1}{\sqrt{M_{s}^{\prime}}} .
$$

This expression should be modified in the same way as Eq. (11) because of the effect of weak Anderson localization.

In 2D, Davy's Eq. (4) becomes

$$
\varepsilon\left\{P_{a}^{\prime}\right\}=\frac{1}{\sqrt{M_{s}}}\left(\left(\frac{3}{2}\right)^{2}-1\right)^{1 / 2} .
$$

One can easily extend Eqs. (B8) and (B9) so as to take account of $1 \mathrm{D}$ modes.

${ }^{1}$ R. H. Lyon, "Statistical analysis of power injection and response averaging in structures and rooms," J. Acoust. Soc. Am. 45, 545-565 (1969).

${ }^{2}$ J. L. Davy, "The relative variance of the transmission function of a reverberation room," J. Sound Vib. 77, 455-479 (1981).

${ }^{3}$ R. L. Weaver, "On the ensemble variance of reverberation room transfer functions, the effect of spectral rigidity," J. Sound Vib. 130, 487-491 (1989).

${ }^{4} \mathrm{R}$. V. Waterhouse, "Estimation of monopole power radiated in a reverberation chamber," J. Acoust. Soc. Am. 64, 1443-1446 (1978).

${ }^{5}$ J. L. Davy, "Improvements to formulae for the ensemble relative variance of random noise in a reverberation room," J. Sound Vib. 115, 145-161 (1987).

${ }^{6}$ T. A. Brody, J. Flores, J. B. French, P. A. Mello, A. Pandey, and S. S. M. Wong, "Random matrix physics: Spectrum and strength fluctuations," Rev. Mod. Phys. 53, 385-479 (1981).

${ }^{7}$ R. S. Langley and V. Cotoni, "The ensemble statistics of the vibrational energy density of a random system subjected to single point harmonic excitation,” J. Acoust. Soc. Am. 118, 3064-3076 (2005).

${ }^{8}$ O. I. Lobkis, R. L. Weaver, and I. Rozhkov, "Power variances and decay curvature in a reverberant system," J. Sound Vib. 237, 281-302 (2000).

${ }^{9}$ J. L. Davy, "The distribution of modal frequencies in a reverberation room," in Proceedings of Inter-Noise 90, Gothenburg, Sweden (1990).

${ }^{10}$ J. L. Davy, "The variance of pure tone reverberant sound power measurements," in Proceedings of the Fifth International Congress on Sound and Vibration, Adelaide, Australia, (1997).

${ }^{11}$ F. Jacobsen, "The diffuse sound field," Ph.D. thesis, The Acoustics Laboratory, Technical University of Denmark, Denmark (1979).

${ }^{12}$ D. Pierce, Acoustics: An Introduction to Its Physical Principles and Applications (McGraw-Hill, New York, 1981), Secs. 6.3 and 6.1.

${ }^{13}$ R. V. Waterhouse, "Statistical properties of reverberant sound fields," J. Acoust. Soc. Am. 43, 1436-1444 (1968).

${ }^{14}$ R. L. Weaver and J. Burkhardt, "Weak Anderson localization and enhanced backscatter in reverberation rooms and quantum dots," J. Acoust. Soc. Am. 96, 3186-3190 (1994).

${ }^{15}$ G. Tanner and N. Søndergaard, "Wave chaos in acoustics and elasticity," J. Phys. A: Math. Theor. 40, R443-R509 (2007).

${ }^{16} \mathrm{P}$. K. Baade and G. C. Maling, "Reverberation room qualification using multitone signals," Noise Control Eng. J. 46, 23-28 (1998).

${ }^{17}$ F. Ihlenburg, Applied Mathematical Sciences (Springer-Verlag, New York, 1998), Vol. 132.

${ }^{18}$ F. Jacobsen, "Sound power determination in reverberation rooms: A normal mode analysis," Ph.D. thesis, The Acoustics Laboratory, Technical University of Denmark, Denmark (1979). 
${ }^{19}$ M. Schröder, "Die statistichen Parameter der Frequenzkurven von grossen Räumen (Statistical parameters of the frequency response curves of large rooms)," Acustica 4, 594-600 (1954).

${ }^{20}$ S. Gade, N. Møller, J. Hald, and L. Alkestrup, "The use of volume velocity source in transfer measurements," in Proceedings of Inter-Noise 2004, Prague, Czech Republic (2004).

${ }^{21}$ Y. Luan and F. Jacobsen, "A method of measuring the Green's function in an enclosure,” J. Acoust. Soc. Am. 123, 4044-4046 (2008).
${ }^{22}$ F. J. Fahy, Sound Intensity, 2nd ed. (E \& FN Spon, London, 1995).

${ }^{23}$ F. Jacobsen, "Sound field indicators: Useful tools," Noise Control Eng. J. 35, 37-46 (1990).

${ }^{24}$ J. Y. Chung and D. A. Blaser, "Transfer function method of measuring in-duct acoustic properties. I. Theory,” J. Acoust. Soc. Am. 68, 907-913 (1980).

${ }^{25}$ P. M. Morse and K. U. Ingard, Theoretical Acoustics (McGraw-Hill, New York, 1968), Sec. 7.3. 